\title{
Parameter Estimation for Automatic Dose Control in Radioscopy
}

\author{
Daniel Keysers, Sami Celik, Henning Braess ${ }^{1}$, Jörg Dahmen, and Hermann Ney \\ Lehrstuhl für Informatik VI, Computer Science Department \\ RWTH Aachen - University of Technology, D-52056 Aachen, Germany \\ Email: keysers@cs.rwth-aachen.de \\ ${ }^{1}$ Philips GmbH Forschungslaboratorien \\ Weißhausstraße 2, D-52056 Aachen, Germany
}

\begin{abstract}
During a medical radioscopic examination, the X-ray dose needs to be adjusted continuously to the body region examined. In current systems, this adjustment is based on the mean grayvalue of the central part of the current image. Basing the control of the X-ray dose on this parameter alone leads to incorrect exposure, if direct radiation enters the central part of the image. We present the application of different regression methods to estimate this parameter more robustly, based on approaches from object classification. Robustness of the estimation is especially important in order to achieve high image quality during the dynamic examination.
\end{abstract}

\section{Introduction}

Radioscopy is a dynamic X-ray examination method which allows the visualization of processes inside the body that are changing with time. These examinations are often combined with the use of a contrast agent, for example in the diagnosis of swallowing movements. In radioscopy, the parameters of the X-ray system need to be adjusted continuously, because they need to match the properties of the regarded tissue, which vary in different regions of the human body. In current systems this adjustment is made on the basis of the mean grayvalue of the central part of the current image. The grayvalue parameter is then used to control the X-ray dose automatically. This procedure leads to incorrect exposure, if direct radiation enters the central part of the image. This problem arises e.g. during barium swall and some vascular examinations. Incorrect exposure is naturally unwanted because it may make the diagnosis more difficult or lead to additional X-ray exposure of the patient if the examination needs to be repeated.

In this work, we present the application of different regression methods for the estimation of the grayvalue parameter using approaches from object classification [1]. The motivation for the use of these methods is that the optimal grayvalue is based on the type of a region of interest. If the method can correctly assign the image to a specific class, the relationship between the grayvalue parameter and the image brightness can be supposed to be approximately linear. Instead of using a two step approach of classification and then parameter 
estimation, we propose to directly view the task as a regression problem. For an introduction to regression see e.g. [2]. Requirements for the estimation procedures are

- robustness, to guarantee a response within the time limits and with noise tolerance,

- proportional response with respect to global image brightness, to allow correct dose control and

- response times below $20 \mathrm{~ms}$, to allow dynamic adjustment of the X-ray dose.

The evaluation of the methods is based on example images from radioscopy which have been labeled with the ideal grayvalue parameter by an expert.

\section{Methods}

The conventional method for the estimation of the grayvalue parameter uses the average of the central $60 \%$ portion of the current image. We propose the use of nearest-neighbor regression, kernel density regression and neural network regression as alternatives. Based on a training set of images $x_{n} \in \mathbb{R}^{D}, n=$ $1, \ldots, N$ for which the optimal grayvalue parameter $y_{n} \in \mathbb{R}, n=1, \ldots, N$ is known (being the mean grayvalue of the object of interest) the three methods estimate the parameter for a new image. That is, the methods can be viewed as functions $f: \mathbb{R}^{D} \rightarrow \mathbb{R}$ to determine the grayvalue, where the free parameters of the functions are determined based on the training set $\left\{\left(x_{n}, y_{n}\right)\right\}$. To reduce the dimensionality of the feature space the images are scaled down from $32 \times 32$ pixels to different sizes, typically $8 \times 8$ pixels, i.e. $D=64$. We use appearance based methods, which means that the grayvalues are used directly as features.

The k-nearest neighbor regression is based on the computation of the $k$ closest training images with respect to a distance function (Euclidean distance here) and returns the average of the optimal parameters of those images.

In kernel density regression, a weighted average of the optimal parameters of the training images is returned, where the weight depends on the value of a kernel function. Here Gaussian kernels were used with multiples of the identity matrix as covariance matrices, scaled with an empirical factor $\alpha$. The resulting estimation function is:

$$
f(x):=\left(\sum_{n=1}^{N} \exp \left(-\frac{\left\|x-x_{n}\right\|^{2}}{2 \sigma^{2} \alpha}\right)\right)^{-1} \sum_{n=1}^{N} y_{n} \exp \left(-\frac{\left\|x-x_{n}\right\|^{2}}{2 \sigma^{2} \alpha}\right)
$$

Finally, in neural network regression the parameter is estimated by an artificial neural net trained on the training images and their optimal parameters. A multi layer perceptron with one hidden layer and sigmoid activation functions was trained using backpropagation in the experiments [3].

The requirement of scaling proportional to total image brightness is achieved by normalizing input and training images and by applying the inverse normalization of the input to the regression output in all three methods. For comparison 

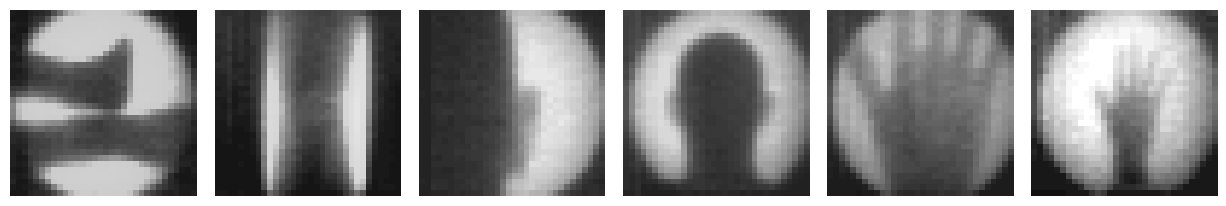

Figure 1. Example images from the database used in the experiments.

we include results obtained by the Philips Research Laboratory using a method based on clustering and segmentation, which performs very well in terms of parameter estimation but is slower and less robust than the regression methods proposed here, as segmentation may fail in some images [4].

\section{$3 \quad$ Experimental results}

We evaluated the approaches using a database of 82 images for which the optimal parameters were determined by an expert. Example images are shown in Figure 1. As the size of this database is quite small, we used leaving-one-out cross-validation to determine the performance, i.e. when estimating the parameter for one image we used the remaining 81 images for training, thus still strictly separating training and test set. The root mean squared error (RMSE) of the estimated parameter with respect to the optimal parameter was used as the performance measure of the algorithms.

$$
\operatorname{RMSE}(f)=\sqrt{\frac{1}{N} \sum_{n=1}^{N}\left\|y_{n}-f\left(x_{n}\right)\right\|^{2}}
$$

Table 1 shows the results for the different methods including the runtime on a standard $800 \mathrm{MHz}$ PC.

It should be noted that the neural net performs best here, but the performance depends strongly on the chosen parameters (number of nodes in the hidden layer and termination criterion for the training). It can be observed that all three methods perform far better than the conventional method (more than $75 \%$ reduction in RMSE) at very low time requirements.

Table 1. Regression results. RMSE over 82 leaving-one-out tests, runtime with respect to one image.

\begin{tabular}{|l|c|c|}
\hline method & RMSE & runtime \\
\hline \hline clustering and segmentation [4] & 1.9 & - \\
\hline mean of 60\% central area & 21.9 & - \\
\hline \hline 1-nearest neighbor & 5.1 & $<1 \mathrm{~ms}$ \\
\hline 3-nearest neighbor & 4.8 & $<1 \mathrm{~ms}$ \\
\hline kernel densities & 4.2 & $<2 \mathrm{~ms}$ \\
\hline neural net & 4.0 & $<0.002 \mathrm{~ms}$ \\
\hline
\end{tabular}




\section{Conclusions}

The presented regression approaches allow a fast and robust estimation of the grayvalue parameter for dose control in medical radioscopy and perform far better than the conventionally used method. They also allow efficient implementation in hardware. The known clustering/segmentation approaches perform still better in terms of RMSE but suffer from instabilities and varying runtimes.

For use in practice the presented methods would use a far larger training set which can be expected to further improve the results. This would increase the runtime for the prototype-based methods (nearest neighbor, kernel densities), but approaches exist to reduce the number of prototypes for these applications (editing and condensing, clustering).

Possible improvements include application of methods known from image object recognition to achieve invariance of the regression result with respect to rotation, scaling and translations of the objects of interest. To evaluate the methods more deeply, a much larger database is also necessary.

\section{Acknowledgement}

We would like to thank Sirko Molau (Lehrstuhl für Informatik VI, RWTH Aachen) for providing the basic neural network implementation.

\section{References}

1. J. Dahmen, D. Keysers, H. Ney, and M. O. Güld. Statistical Image Object Recognition using Mixture Densities. Journal of Mathematical Imaging and Vision, 14(3):285-296, May 2001.

2. V. Cherkassky and F. Mulier. Learning from Data. Wiley, New York, 1998.

3. C. M. Bishop. Neural Networks for Pattern Recognition. Oxford Univ. Press, 1996.

4. H. Braess and G. Schmitz. Philips Research. Personal communication, 2001. 